\title{
Accompagner la démarche de management stratégique de l'exploitation agricole
}

A methodological framework to help farmers to implement strategic farm management

Mathieu Capitaine, Ambroise Garnier, Philippe Jeanneaux, Frank Pervanchon, Yann Chabin, Nathalie Bletterie, Bertrand de Torcy et Hugues de Framond

\section{OpenEdition}

\section{Journals}

Édition électronique

URL : http://journals.openedition.org/economierurale/4118

DOI : $10.4000 /$ economierurale. 4118

ISSN : 2105-2581

Éditeur

Société Française d'Économie Rurale (SFER)

Édition imprimée

Date de publication : 15 octobre 2013

Pagination : 75-90

ISSN : 0013-0559

Référence électronique

Mathieu Capitaine, Ambroise Garnier, Philippe Jeanneaux, Frank Pervanchon, Yann Chabin, Nathalie Bletterie, Bertrand de Torcy et Hugues de Framond, « Accompagner la démarche de management stratégique de l'exploitation agricole », Économie rurale [En ligne], 337 | septembre-octobre 2013, mis en ligne le 15 octobre 2015, consulté le 30 avril 2019. URL : http://journals.openedition.org/ economierurale/4118; DOI : 10.4000/economierurale.4118 


\title{
Accompagner la démarche de management stratégique de l'exploitation agricole
}

\author{
Mathieu CAPITAINE • Ambroise GARNIER • Philippe JEANNEAUX • VetAgro Sup, \\ Clermont Université, UMR Métafort, Lempdes \\ Frank PERVANCHON • Resolia, APCA, Paris \\ Yann CHABIN • Cregor, Université Montpellier II \\ Nathalie BLETTERIE • SupAgro, Florac \\ Bertrand de TORCY • Trame, Paris \\ Hugues de FRAMOND • Draaf-SRFD Auvergne, Lempdes
}

\begin{abstract}
Développer la démarche de management stratégique dans les exploitations agricoles est une façon d'améliorer leurs performances dans le contexte actuel de l'agriculture. Les auteurs proposent un itinéraire méthodologique qui vise à accompagner les exploitants agricoles dans la construction de stratégies et dans leur traduction opérationnelle. Structuré en trois étapes, il aide à organiser la réflexion autour de la stratégie, puis à la formaliser, pour ensuite la piloter grâce à un tableau de bord stratégique. Le lien entre ces trois étapes est assuré par la construction d'une carte causale. Cet itinéraire a été construit dans le cadre du programme de recherche-action PerfEA avec des établissements publics locaux d'enseignement agricole. L'itinéraire s'adresse à tout type d'exploitation agricole et sa mise en œuvre nécessite la présence d'un conseiller.
\end{abstract}

MOTS-CLÉS : agriculture, stratégie, conseil, carte causale, tableau de bord

\section{A methodological framework to help farmers to implement strategic farm management}

Strategic management is a relevant approach to support farmers in managing their farm and in coping with the current agricultural challenges. In this article we propose a methodological route which aims at enabling farmers to design their strategy and to implement them. The route is organized along three steps: it helps to organize the strategic thinking; to formalize the strategy by defining priorities and strategic lines; to draw up a strategic monitoring plan (the balanced scorecard). The link between the different steps is provided by the design of a causal mapping. This methodological route was built within an action research project entitled PerfEA (Global Performance for Farm) including farms belonging to public training schools. It suits all kind of farms and requires an agricultural adviser to accompany farmers in the design process of the strategy and its implementation. (JEL: L21; Q13).

KEYWORDS: agriculture, strategy, advice, causal mapping, balanced scorecard

e contexte de la production agricole ap-paraît aujourd'hui plus complexe. Tout d'abord, en raison de la baisse tendancielle des prix de nombreux produits agricoles, les structures de production ont évolué pour contrecarrer la baisse du revenu par exploitation en cherchant notamment à réaliser des gains de productivité. La taille des exploitations a augmenté et s'est souvent accompagnée d'une saturation de la capacité de travail (Dedieu et al., 1999 ; Mignolet et al., 1998), avec une part du travail familial en diminution (Agreste, 2011). Les exploitations agricoles sont de plus en plus intenses en capital et leur nombre sous forme sociétaire continue de 
progresser (Rattin, 2007). Les plus grosses exploitations ont tendance à se spécialiser, alors que certaines développent de nouvelles activités en vue de se différencier (agritourisme, services environnementaux, etc.) (Agreste, 2011). Une exploitation sur cinq commercialise en circuit court, plus particulièrement dans les exploitations de petite taille économique (Agreste, 2011). Les exploitations agricoles s'éloignent petit à petit du modèle de l'exploitation agricole familiale (Laurent, 2000 ; Séronie et al., 2007).

Au gré des crises environnementales, sanitaires et économiques, l'encadrement par les pouvoirs publics des activités agricoles s'est intensifié. Les exploitations sont confrontées à des modifications de la Politique agricole commune (PAC) avec la réorientation des subventions du premier vers le second pilier, la disparition progressive des mécanismes de soutien et de régulation des marchés, et la montée en puissance des exigences de respect des bonnes conditions agricoles et environnementales. L'encadrement des exploitations se renforce également par les acteurs privés de l'aval, notamment avec l'augmentation des relations contractuelles entre producteurs et industriels de l'agroalimentaire, et le développement de cahiers des charges et d'exigences spécifiques. Les agriculteurs sont dans un contexte de plus en plus concurrentiel et ainsi moins protégés des risques de marché. À l'instar de ce qui s'est produit dans les secteurs de l'industrie et des services, les exploitations agricoles vont devoir améliorer au fur et à mesure leurs performances techniques, économiques, environnementales et leur responsabilité sociétale pour maintenir leur activité.

Ce contexte concurrentiel et complexe peut poser des questions aux agriculteurs. Comment rester présent sur le marché et se rendre incontournable ? Comment obtenir et conserver un avantage concurrentiel ?
Comment intégrer les évolutions de l'environnement de l'exploitation dans la conduite des activités ? Comment développer une capacité à faire face aux aléas tout en maintenant ou améliorant le niveau de performance de l'exploitation ? Ces questions se répercutent sur les conseillers agricoles et les conduisent à s'interroger sur leurs modes d'intervention auprès des agriculteurs.

Ces questions ne trouvent pas de réponses satisfaisantes avec les conceptions classiques de la gestion de l'exploitation agricole héritées de Chombard de Lauwe et al. (1969). Comparer ses résultats comptables aux meilleurs par l'analyse de groupe en identifiant les leviers clés censés permettre de les rejoindre n'est plus une approche pertinente. On sait depuis les travaux de Brossier et al. (1988) que les résultats comptables ne correspondent pas à la vision qu'a l'agriculteur de son activité et qu'ils servent peu pour l'aide à la décision.

Ces questions, également posées à l'entreprise en général, sont classiquement traitées par les spécialistes de la gestion d'entreprise en termes de stratégie et de management stratégique de la firme. Le management stratégique consiste à organiser dans la durée la nécessaire adéquation entre des exigences de l'environnement et les capacités de l'entreprise pour atteindre ses objectifs. Le management stratégique est donc le processus au travers duquel les stratégies sont identifiées puis mises en œuvre (Barney, 1991). Nous faisons le postulat que mobiliser ces approches dans le cadre de l'exploitation agricole devrait l'aider à se positionner et à maintenir son activité. Ce postulat est soutenu par trois arguments :

- Les démarches de management stratégique sont maintenant fortement développées dans le secteur de l'industrie et des services (tant dans les grandes entreprises que dans les PME) et ont fait 
preuve de leur pertinence (Lérat-Pytlak, 2002). Elles sont le fondement d'un certain nombre de dispositifs de certification du management de la qualité (série des normes ISO 9000 et ISO 14000).

- Ces démarches sont déjà mobilisées dans des exploitations agricoles (qualifiées dans cet article de privées) gérées par des agriculteurs professionnels (Mazé et al., 2000 ; Trémolet, 2008) ainsi que dans des exploitations agricoles d'établissement d'enseignement public (Mellet, 2007). Elles sont également appliquées dans des structures coopératives assurant la collecte, la transformation et la commercialisation de produits agricoles (Chabin, 2008).

- D'ores et déjà des dispositifs de formation, à destination des agriculteurs, sur la stratégie d'entreprise sont proposés (Goulet et al., 2008 ; Pervanchon et al., 2007).

Cependant, ces approches, même si elles existent, restent encore peu développées dans les exploitations agricoles. Les expériences citées ci-dessus sont emblématiques et exceptionnelles. Bien souvent, si des démarches intégrant le management stratégique sont présentes, elles ne sont pas organisées et assez peu formalisées.

Ce constat vaut également pour les travaux scientifiques français en sciences de gestion. Rares sont les travaux originaux en gestion de l'exploitation qui traitent de management stratégique (Jeanneaux et al., 2012). Ils se limitent aux travaux de quelques chercheurs qui ont proposé dans le milieu des années 1990 une mise en œuvre de la démarche de management stratégique à l'exploitation (Guichard et al., 1994 ; Hémidy et al., 1996 ; Hémidy et al., 1993 ; Hémidy et al., 1994), alors qu'existe une littérature assez abondante en langue anglaise sur la démarche de management stratégique adaptée à l'exploitation agricole ; une quinzaine d'ouvrages depuis le début des années 1980 traite du farm management comme ceux de Barnard et al. (1979), de Casavant et al. (1999), de Kay (1986), d'Olson (2004), ou encore de Turner et al. (1998) pour ne citer que les plus connus.

Enfin, la démarche de management stratégique nécessite une prise de recul qui peut être réalisée par les conseillers en agriculture. Cependant, ces derniers ne sont pas tous familiers des démarches d'accompagnement. Ils sont pour certains encore très marqués par l'approche descendante du conseiller qui vient avec son expertise régler les problèmes de l'agriculteur. Or ils interviennent de plus en plus dans des contextes complexes qui demandent une autre expertise visant à interroger la stratégie globale de l'exploitation agricole. Force est de constater que le nombre de conseillers en agriculture formé à mobiliser les approches stratégiques est faible (Vernerey, 2007). De plus, peu d'outils sont disponibles pour accompagner les agriculteurs afin de travailler autour :

- de la compréhension des perspectives agricoles, de l'analyse du contexte et des motivations de l'agriculteur,

- de la formalisation de stratégies,

- de l'accompagnement au pilotage stratégique et à l'évaluation des performances obtenues.

Pour développer le management stratégique en exploitation agricole, un des enjeux est donc de fournir les capacités aux conseillers d'initier et d'accompagner les agriculteurs dans cette démarche.

Nous présentons l'architecture d'un itinéraire méthodologique pour aider à la mise en œuvre du management stratégique dans les exploitations agricoles. Avant de le présenter, nous aborderons le cadre d'analyse mobilisé (2). Nous exposerons ensuite le dispositif de recherche dont il est issu (3), puis ses principales étapes (4). Dans un dernier temps, nous reviendrons sur les conditions qu'un tel travail nécessite pour sa mise en œuvre en exploitation agricole (5). 


\section{Cadre d'analyse de la démarche de management stratégique}

Centrée sur l'analyse que l'agriculteur fait de sa situation et de l'évolution de son environnement, la démarche de management stratégique doit lui permettre d'établir un projet d'exploitation et de le mettre en œuvre. Il s'agit pour l'agriculteur de pouvoir se construire une stratégie et se doter d'éléments afin de faire des choix et de hiérarchiser ses différents objectifs. L'itinéraire méthodologique proposé doit permettre d'expliciter et de formaliser la représentation de la stratégie et du processus pour la mettre en œuvre et la piloter.

Nous avons choisi de nous appuyer sur le cadre, bien connu en sciences de gestion, du management stratégique qui recouvre quatre étapes principales représentées par un cycle d'amélioration continue (cycle de Deming ou Shewhart) (Shewhart, 1989). (i) La planification de la stratégie passe par la définition et la formalisation d'une politique générale, déclinée ensuite en objectifs stratégiques et en orientations d'action, puis par la construction d'un plan d'action. Dans ce cadre, nous considérons l'exploitation comme un système piloté et finalisé (Marshall et al., 1994) ouvert sur le monde (l'environnement, les parties prenantes) avec l'idée d'ouvrir le débat pour aider à la découverte de solutions partagées. (ii) La mise en œuvre du plan d'action permet de progresser de la situation actuelle vers la situation projetée. (iii) Le contrôle des résultats obtenus cherche à évaluer le plan d'action et l'atteinte des performances souhaitées. (iv) La réinterrogation de la stratégie au regard de l'évaluation doit favoriser, le cas échéant, l'adaptation des objectifs stratégiques et la modification du plan d'action.

L'amélioration continue vise à aider l'agriculteur à traiter la complexité en situation de pilotage de l'exploitation. Il s'agit de dépasser trois incertitudes liées (i) à l'impossibilité de raisonner en termes de causalité linéaire ; (ii) au fait que le résultat d'une action est en partie déterminé par l'environnement ; (iii) à l'interdépendance des parties qui constituent l'exploitation (Thiétart, 2004). Pour ce faire, le pilote du système doit avancer à petits pas et revoir régulièrement le déroulement de ses actions pour les réactualiser en vue de s'adapter. En gardant le sens global de l'action et des objectifs à atteindre, aucune action mise en œuvre est irréversible. La démarche doit permettre de garder la mémoire des expérimentations, des réussites et des échecs pour réagir en se constituant un répertoire d'expériences et une capacité de réaction aux changements et à l'incertitude.

Nous avons également fait le choix de ne pas produire de préconisations à caractère normatif. L'objectif est de proposer un cadre méthodologique qui aide l'agriculteur à construire une stratégie non normative et située ; non normative car propre à chaque exploitation et à chaque projet et située car adaptée aux spécificités du contexte de l'exploitation.

C'est d'une certaine manière une exigence en contradiction avec la conception normative de la gestion de l'exploitation distillée par Chombart de Lauwe et al. (1969). Les bonnes recettes des uns ne sont pas forcément les bonnes recettes des autres. Gérer ce n'est pas forcément établir un diagnostic économique et financier fondé sur des données comptables que l'on pourra comparer pour in fine identifier les leviers sur lesquels agir pour augmenter les profits. D’une part, les données comptables demandent de l'expertise et mobilisent trop souvent des indicateurs de performance datés peu pertinents pour l'agriculteur (Brossier et al., 1997). L'agriculteur ne peut pas évaluer ses choix avec des données qui n'ont pas de sens dans son système d'information et dans son dispositif de contrôle des performances. D'autre part, l'agriculteur n'est pas forcément 
maximisateur de profit. Il peut mettre en cause les schémas dominants, proposer des alternatives, s'inscrire dans d'autres logiques à l'instar du comportement des autres agents économiques (Simon, 1982).

Enfin, sur le plan épistémologique, nous retenons l'idée que la performance est un construit social qui puise ses fondements dans les représentations individuelles que chacun développe de son organisation et de sa stratégie (Chabin, 2001). Notre approche se réclame du paradigme constructiviste en sciences sociales. Il nous fournit, pour cette recherche sur le management stratégique, un cadre de modélisation adapté pour aborder la complexité des organisations sociales en donnant des repères heuristiques pour susciter le questionnement, éclairer des situations problématiques et/ou stimuler l'action créative (Avenier et al., 2008). Ainsi, comme le propose Simon (2004), les membres d'une organisation essaient simultanément d'adapter leur environnement à leurs désirs et, dans la mesure où en général ils n'y parviennent pas totalement, de s'adapter à leur environnement. Par conséquent, sur la base de ces considérations, définir et piloter une stratégie, c'est faire évoluer sa représentation de la performance au sein de l'exploitation.

\section{Programme de recherche-action avec des établissements publics d'enseignement agricole et des exploitations privées}

L'itinéraire méthodologique présenté a été construit au cours du programme de recherche-action PerfEA (2009-2012), dont les objectifs étaient de proposer et tester des méthodes et des outils pour concevoir une démarche permettant de développer le management stratégique dans les exploitations agricoles. Nous avons conduit ce programme en deux temps principaux.

- Le premier temps a porté sur la coconstruction de l'itinéraire méthodologique.
Nous avons choisi de travailler avec sept exploitations d'établissements publics locaux d'enseignement et de formation professionnelle agricoles du Massif central. Le ministère en charge de l'Agriculture demande à ces exploitations agricoles de définir tous les cinq ans un projet d'exploitation qui permet à l'administration d'avoir une idée de la situation et de l'orientation de l'exploitation, sans toutefois que soient approfondies les questions de la mise en œuvre et de la conduite du projet. La rédaction du projet de l'exploitation contient différents volets (techniques, économiques, pédagogiques). Ces exploitations ont par ailleurs une mission d'expérimentation et de développement à remplir, cadre favorable sur lequel nous nous sommes appuyés. Elles ont permis d'engager un premier travail de conception qui aurait été peu supportable par des exploitations privées (prise de risque et temps de conception à libérer), tout en gardant un souci d'opérationnalité lié à l'exigence d'aboutir à un itinéraire méthodologique utilisable. Ces exploitations sont des structures complexes. En plus de la nécessité d'assurer leur équilibre économique, elles doivent être un support pédagogique performant. Elles sont généralement dépendantes du contexte institutionnel et politique agricole départemental et régional et se trouvent parfois au centre de jeux d'influence. Les acteurs associés à la conduite de l'exploitation sont souvent nombreux et ont des fonctions et des implications différentes (directeur d'exploitation, directeur d'établissement, salariés, enseignants, apprenants, membres des conseils constitutifs de l'établissement). De plus, la gouvernance du projet est rendue délicate par de fréquents changements des acteurs. Construire un projet pour piloter ce type d'exploitation demande donc une réelle réflexion stratégique. Dans les faits, les responsables 
de ces exploitations se trouvent devant des difficultés méthodologiques pour la mener à bien. Ils s'interrogent sur la façon d'assurer la mobilisation des collectifs, l'animation des réflexions, la facilitation des discussions, la primauté des objectifs et des actions, la définition des dispositifs d'évaluation des résultats tout en devant assurer au quotidien le fonctionnement de l'organisation. Sur la base des délimitations méthodologiques proposées par David (2000), notre démarche a consisté à construire de la connaissance en cherchant à utiliser les situations empiriques rencontrées et étudiées dans les sept exploitations supports, pour discuter et amender les propositions théoriques sur lesquelles nous nous sommes appuyés. Nous avons constitué un groupe scientifique et technique composé de représentants du développement agricole (Trame, Résolia), du conseil en management (Dycia), de l'enseignement supérieur (VetAgro Sup, SupAgro Florac, Université Montpellier 2), de l'enseignement technique agricole (DRAAF-SRFD Auvergne). Ce groupe a défini le cadre théorique de l'itinéraire méthodologique. Nous avons accompagné durant trois ans chacune des exploitations agricoles engagées et fait travailler leurs équipes sur et avec les outils permettant de constituer l'itinéraire méthodologique. Un ingénieur d'études dédié au projet a joué le rôle de l'accompagnateur pour mettre en œuvre l'itinéraire méthodologique dans un cadre de coconstruction. Les responsables des sept exploitations ont été réunis trois fois par an pour tirer collectivement des enseignements de leurs expériences afin d'amender et stabiliser l'itinéraire méthodologique. Les exploitations participantes ont des productions différentes (bovin lait, élevage naisseur engraisseur bovin-ovinporcin, grandes cultures, pisciculture) et évoluent chacune dans un territoire aux enjeux particuliers. Cette diversité de situations nous a donné un cadre pertinent pour tester la généricité de l'itinéraire méthodologique conformément à notre objectif de ne pas produire des préconisations à caractère normatif, mais bien de travailler sur un itinéraire méthodologique qui aide à construire une stratégie adaptée et située.

- Le deuxième temps a visé à tester l'itinéraire méthodologique sur huit exploitations agricoles privées. Elles ont été également retenues pour la diversité de leurs situations en termes de production, de statut juridique, de position dans leur cycle de vie, ou encore de localisation géographique. Nous avons pu ainsi compléter l'itinéraire, puis valider sa faisabilité et sa pertinence pour ce type d'exploitation.

\section{Itinéraire méthodologique de la démarche de management stratégique}

Reprenant le principe de l'amélioration continue, l'itinéraire méthodologique est organisé en trois étapes principales $(f-$ gure 1). Son originalité est qu'il s'appuie, d'une part, sur un outil particulier, la carte causale, pour assurer le lien entre les trois étapes et garantir une capitalisation et une mobilisation des informations produites. Il propose, d'autre part, des outils de pilotage stratégique et notamment le tableau de bord stratégique équilibré au sens du «balanced scorecard » proposé par Kaplan et Norton (Kaplan et al., 1996) et enrichi par d'autres comme Lorino (2001). L'association de la carte causale et du tableau de bord avec des outils d'animation et de réflexion collective dans un itinéraire méthodologique constitue une innovation pour élaborer une stratégie et aider à sa mise en œuvre et à son pilotage. La démarche stratégique proposée doit permettre d'établir une stratégie pour un horizon de temps de 3 à 5 ans. 


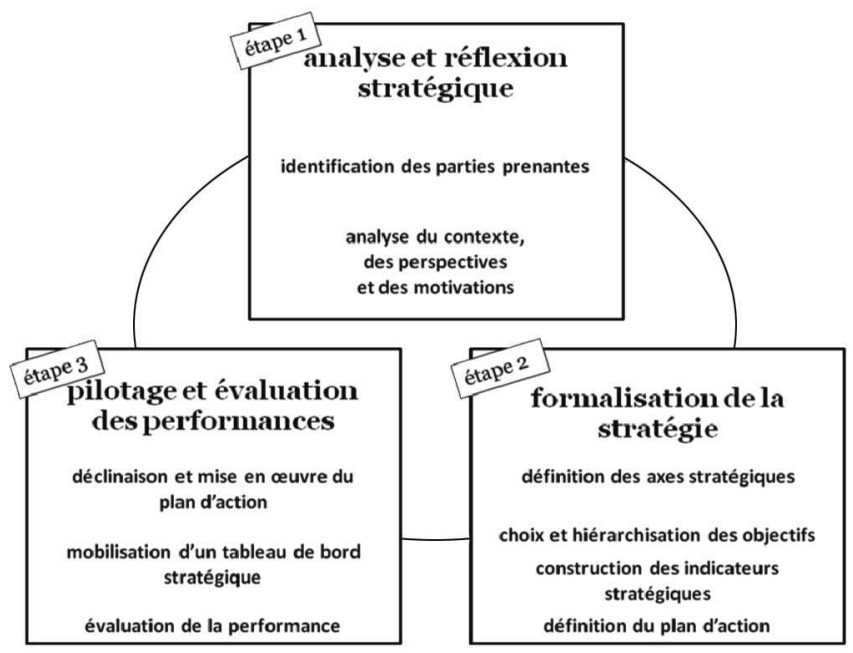

Note : Chaque carré représente une étape et indique les objectifs qui lui sont associés.

\section{La carte causale, lien entre les trois étapes de l'itinéraire}

Le lien entre les trois étapes de l'itinéraire méthodologique est assuré en mobilisant un outil qui donne à voir les représentations qu'ont les agriculteurs de leur exploitation : la carte causale. Elle est définie par Eden (1988) comme : «Un modèle conçu pour représenter la façon dont une personne définit un problème particulier. $\mathrm{Ce}$ n'est pas un modèle général de la façon de penser de quelqu'un [...]. » Sur le plan pratique, c'est une représentation graphique qui articule des entités nommées (idées, enchaînement d'idées) en fonction de liens de causalité, repérables dans le discours, formant ainsi des chaînes de causalité complexes car en interrelation (figure 2). Pour construire les chaînes de causalité, nous partons de l'hypothèse que les représentations des agriculteurs sur le fonctionnement de leur exploitation sont formulées en termes de lien de cause à effet : une variable initiale est une condition préalable à une variable finale. Par exemple : «Avoir une ration à base de fourrage sec permet de respecter le cahier des charges de l'AOP, qui permet de produire du lait $A O P$, qui permet d'avoir un prix de lait supérieur au lait standard produit dans la zone AOP... » La relation d'influence porte sur le pourquoi de l'effet et suggère une réponse renvoyant aux causes qui le précèdent et commençant par parce que (Cossette, 1994). L'utilisation de la carte causale pour explorer les structures cognitives dans une organisation est désormais largement répandue dans les recherches en gestion (Huff, 1990 ; Laukkanen, 1998). Particulièrement adaptée aux démarches stratégiques (Cossette, 2003 ; Eden, 1988), elle permet de formaliser et de donner à voir les représentations des individus et des collectifs. Le type de carte causale que nous avons réalisé est une représentation à l'échelle d'un collectif. Il s'agit d'une carte dite stratégique qui réunit les visions de plusieurs membres d'une organisation. 
Elle se distingue de la catégorie des cartes dites composites qui résultent de l'agrégation de cartes individuelles.

Par sa construction, la carte causale permet de représenter la complexité sans l'évacuer (Axelrod, 1976) et d'exposer les représentations des acteurs conformément au cadre épistémologique du constructivisme retenu et présenté. Elle aide les responsables de l'exploitation à acquérir une plus grande compréhension de leur situation, à mieux formuler les problèmes et leurs interrelations. Elle permet de développer des idées nouvelles sur les orientations de l'exploitation afin d'élargir les choix possibles. C'est à la fois un outil d'aide à la communication et un outil d'analyse (Cossette, 2003). Elle illustre des relations de causalité et une partie des raisonnements à l'origine de décisions. Elle est un support de médiation qui clarifie la réflexion et la prise de décision. Elle peut permettre à un collectif de s'accorder sur une stratégie et d'en avoir une vision partagée (Eden, 1988). C'est un outil qui, à notre connaissance, n'a pas été utilisé en France pour définir les choix stratégiques des exploitations agricoles. Elle a toutefois été utilisée pour étudier les représentations cognitives de différents acteurs dans différents contextes, dont certains, proches de ceux étudiés ici, comme la performance de caves coopératives viticoles (Chabin, 2008) ou encore la perception du socio-écosystème de l'exploitation agricole par des agriculteurs (Fairweather, 2010).

La carte causale est construite avec un logiciel de modélisation cognitive (Logiciel Decision Explorer de la société Banxia) qui nous permet de réaliser des analyses topographiques et mathématiques de données textuelles utiles à la construction de la stratégie. Les traitements mathématiques permettent de donner un poids aux unités d'information en fonction de leur lien aux autres unités d'informations, et non pas en fonction de l'importance perçue attribuée par les fréquences.
L'analyse topographique s'appuie sur un travail préalable de hiérarchisation et de positionnement des entités selon leur place dans les chaînes de causalité. La carte causale permet d'identifier de multiples chaînages (plus ou moins interdépendants, plus ou moins concurrents, plus ou moins contradictoires, plus ou moins importants) qui concourent à la réalisation d'un même but. Sont également rendues visibles des boucles d'amélioration ou de dégradation sur lesquelles l'attention sera portée.

L'analyse mathématique s'intéresse au nombre des liens et à la longueur des chaînes qui relient chaque entité. (i) Elle permet tout d'abord de mettre en évidence des groupes d'éléments fortement liés les uns aux autres et ayant une cohérence propre et en même temps à identifier dans la carte des groupes de concepts mutuellement exclusifs, groupes d'idées faiblement dépendant les uns des autres. Il s'agit d'un algorithme de « clusterisation » à base de marches aléatoires. Par exemple, sur les exploitations des établissements d'enseignement agricole ressortent des ensembles cohérents autour de buts économiques, pédagogiques et d'ancrage territorial qui permettent d'identifier les grands domaines de performance de l'exploitation, et en les croisant, de définir les axes stratégiques. (ii) L'analyse permet ensuite de repérer les entités les plus structurantes de la carte, car reliées directement ou indirectement à beaucoup d'autres entités et insérées dans des chaînes complexes : ce sont des centralités. L'algorithme utilisé par le logiciel consiste à calculer des moyennes pondérées des nombres de liens entre entité et de leur distance à chaque lien. Ces entités sont essentielles à la stratégie, parce qu' au cœur de multiples chaînes de causalité. Elles révèlent les leviers possibles pour atteindre les buts de l'exploitation. La définition de la stratégie, la construction du tableau de bord stratégique, puis du plan d'action, s'appuient sur les résultats de ces analyses. 
RECHERCHES

\section{Trois étapes successives}

La première étape (environ trois demijournées de travail) s'appuie sur l'analyse des perspectives agricoles, du contexte et des motivations des parties prenantes de la stratégie (agriculteurs ou directeurs d'exploitation, salariés...). Cette étape doit permettre, tout d'abord, de définir à l'aide du jeu des frontières les contours de l'exploitation et ses relations internes et externes (Charbonnier et al., 2009). Dans le jeu des frontières, la notion de frontière est utilisée pour amener les participants à positionner les éléments qui sont à l'intérieur ou ceux qui sont à l'extérieur de l'organisation et qui auront une influence sur la stratégie. L'existence et la nature des flux entre ces éléments, et les collaborations sont également repérées. Cette étape aide à identifier les parties prenantes potentielles de la réflexion, dont la présence va conditionner le contenu des échanges. Ensuite, la réflexion porte sur les réussites et les échecs de l'exploitation, l'expression de missions, de valeurs et d'une vision de l'exploitation par la projection dans l'avenir. Les analyses du fonctionnement interne de l'exploitation et de ses relations à l'environnement sont aussi des passages proposés dans l'itinéraire méthodologique. Chacun de ces points est instruit dans l'itinéraire méthodologique à l'aide d'outils d'animation et de créativité (photo-langage, remue-méninges, groupe de discussion) (Jérome, 2001) (figure 2, 1.1). Une réflexion particulière sur les relations du système à son environnement est proposée (Bossel, 1999). Toutes les informations produites sont retranscrites sous la forme d'une carte causale par l'accompagnateur-animateur du travail (figure 2, 1.2). Chaque carte causale produite est soumise aux parties prenantes pour valider sa conformité avec leur vision de la stratégie de l'exploitation. Les traitements de base de la carte permettent d'identifier les buts, les entités de base, les entités centrales et également les grands domaines de performance de l'exploitation.

Figure 2. Le processus de la première étape

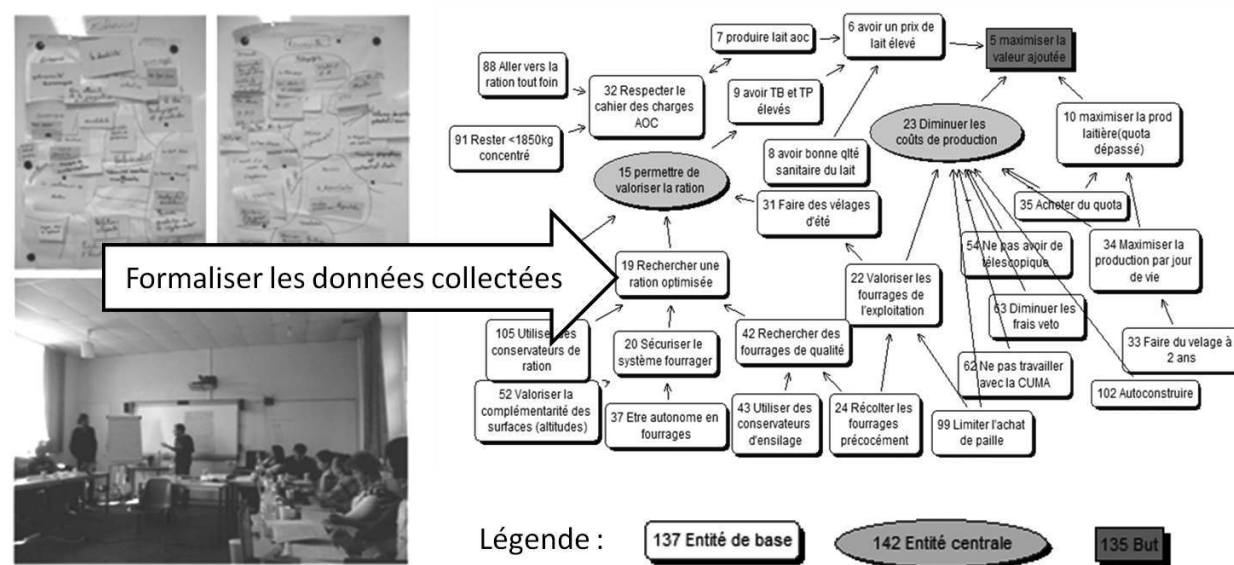

1.1 ateliers pour débattre des missions, des valeurs et des projets de l'exploitation
1.2 une carte causale pour formaliser les représentations collectives de l'exploitation 


\section{Management stratégique des exploitations agricoles}

La deuxième étape (une demi-journée de travail) mobilise l'ensemble des informations produites lors de la première étape. Elle vise à formaliser la stratégie. L'accompagnateur s'appuie sur l'analyse de la dernière carte produite pour, avec les parties prenantes, définir des axes stratégiques. Ils doivent exprimer les orientations majeures de l'exploitation. Pour chaque axe, sont identifiées les entités qui constituent des objectifs stratégiques ; entités qui sont centrales pour l'atteinte des buts de l'exploitation et devront être pilotées (figure 3, 2.1). Pour ce faire, elles sont traduites en indicateurs stratégiques. Ils doivent être pertinents, fiables, facilement mesurables. Ils s'appuient sur des valeurs seuils pour donner un sens à la mesure. Ils constituent un tableau de bord stratégique, principal outil du management stratégique (Chabin, 2008) (figure 3, 2.2).

L'attention des acteurs en charge du pilotage de la stratégie étant une ressource rare (Simon, 1982), le tableau de bord doit être conçu de manière à focaliser leur vigilance et leur permettre d'avoir une vision globale et intelligible de leur stratégie.
Pour ce faire, les indicateurs stratégiques retenus sont limités en nombre (sept à dix).

Le tableau de bord est équilibré ( $B a$ lanced Scorecard) dans le sens où il est construit avec l'ambition d'être multidimensionnel en intégrant des critères financiers et non financiers, de court et de long terme, qualitatifs et quantitatifs, rétrospectifs et prospectifs. À notre connaissance, il n'a été appliqué à des exploitations agricoles qu' aux Pays-Bas (Noell et al., 2002). La cohérence d'ensemble des indicateurs est construite par les participants à la réflexion stratégique. Ceux-ci choisissent les indicateurs en fonction de leur position dans la carte, de leur statut donné par le traitement statistique (but, centralité), de leur présence dans chacun des domaines de performances et des axes stratégiques. Les participants évitent ainsi de retenir des indicateurs redondants. Les traitements proposés aident les participants à sélectionner les entités clés sur lesquelles ils peuvent mettre des indicateurs, soit parce qu'elles ont des effets majeurs dans une chaîne de causalité, soit parce qu'elles sont des centralités, soit encore parce qu'elles

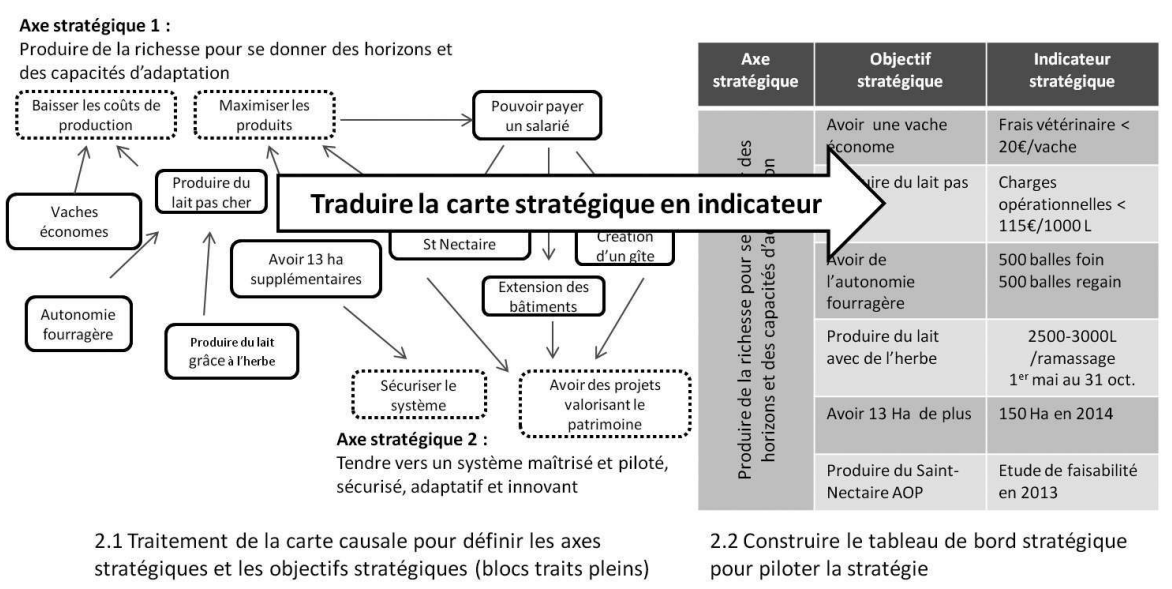

Source : les auteurs. 


\begin{tabular}{|c|c|c|c|c|c|}
\hline \multicolumn{4}{|c|}{ Tableau de bord stratégique } & \multicolumn{2}{|c|}{ Plan d'action } \\
\hline $\begin{array}{l}\text { Objectif } \\
\text { stratégique }\end{array}$ & Indicateur & Valeur de l'indicateur & $\begin{array}{l}\text { Fréquence de } \\
\text { contrôle }\end{array}$ & actions & Moyens d'action \\
\hline \multirow{3}{*}{$\begin{array}{l}\text { Produire du } \\
\text { lait pas } \\
\text { cher }\end{array}$} & \multirow{3}{*}{$\begin{array}{c}\text { Charges } \\
\text { opérationnelles } \\
/ 1000 \mathrm{~L}\end{array}$} & \multicolumn{3}{|c|}{ Créer un plan d'action et mettre en œuvre } & Re-semer des prairies avec des \\
\hline & & \multirow{2}{*}{$\begin{array}{c}\text { inférieur ou égale à } \\
115 € / 1000 \mathrm{~L} \\
\text { (niveau de 2010) }\end{array}$} & \multirow[t]{2}{*}{$\begin{array}{l}\text { Chaque } \\
\text { campagne } \\
\text { laitière }\end{array}$} & $\begin{array}{l}\text { Réduire la fertilisation minérale en } \\
\text { valorisant les engrais de ferme }\end{array}$ & Composter le fumier \\
\hline & & & & $\begin{array}{l}\text { Limiter les concentrés/Vache } \\
\text { Laitière }\end{array}$ & $\begin{array}{l}\text { Définir une ration avec } 700 \mathrm{~kg} \text { de } \\
\text { concentré / vache/an }\end{array}$ \\
\hline
\end{tabular}

3. Passage du tableau de bord au plan d'action pour mettre en œuvre la stratégie

Source : les auteurs.

sont des buts de l'exploitation qu'il faut contrôler et piloter.

La troisième étape concerne le pilotage stratégique et l'évaluation des performances. C'est la phase de déclinaison et de mobilisation du tableau de bord. Un plan d'action connexe au tableau de bord est construit pour guider l'action des responsables de la mise en œuvre de la stratégie (figure 4). Sa définition repose sur les indicateurs stratégiques comme cible à atteindre pour la définition du plan d'action (Kaplan et al., 2004). L'indicateur n'a en effet d'utilité que relativement à une action à piloter (Lorino, 2001). Le plan d'action a deux fonctions majeures : c'est un outil de pilotage qui peut être révisé régulièrement pour adapter les décisions aux aléas ; c'est un outil d'animation du collectif en charge de la mise en œuvre de la stratégie. Les actions à mettre en œuvre et les moyens à mobiliser sont définis par les parties prenantes de la stratégie. Ce plan d'action permet d'organiser la délégation du pilotage des actions et favorise ainsi la responsabilisation de tous les acteurs de la démarche. Sa forme et son degré de précision sont laissés à la discrétion des parties prenantes en charge de la mise en œuvre. Cette étape demande une journée de travail pour le groupe, puis des demi-journées d'animation les années suivantes nécessaires pour donner de la visibilité, s'adapter aux changements et communiquer sur l'avancement de la démarche stratégique.

\section{Intérêts, limites et conditions de mise en œuvre de la démarche}

Par construction, l'itinéraire méthodologique est adaptable à de nombreuses situations. Nous l'avons testé dans deux situations différentes. Premièrement, avec des exploitations agricoles privées sans rencontrer de difficultés majeures. Deuxièmement, avec un groupe de quatre exploitations agricoles privées qui se sont réunies à six reprises pour réaliser chacune leur réflexion stratégique dans un cadre collectif d'échange entre pairs.

Sur un plan pragmatique, aux dires des participants (une vingtaine d'accompagnements réalisés), la mise en œuvre de l'itinéraire méthodologique est appréciée même si elle peut entraîner certaines difficultés (temps à consacrer, tensions pouvant être exacerbées). Les participants disent qu' "avoir un animateur extérieur, ne pas se sentir jugé, c'est appréciable». La méthode permet de «se poser, prendre du recul et de la hauteur; elle nous remet au cour de l'exploitation et nous rend plus autonome, plus responsable ». D'autres ajoutent : «La méthode est positive, ne cassant pas l'ancien et le faisant évoluer»; «on a pu faire un travail partagé »; " la carte causale, c'est rigolo !». Enfin, concernant le pilotage «on s'est fixé un petit nombre d'objectifs réalisables, fédérateurs et motivants pour les équipes ». 


\section{Management stratégique des exploitations agricoles}

Sur un plan théorique, nous ne sommes pas sans savoir que les approches de planification stratégique font l'objet de critiques. (i) Mintzberg (1994) leur reproche d'être analytiques, descendantes, focalisées sur la mesure quantitative de ratios souvent financiers, en faisant perdre de vue la vision systémique de la stratégie par le contrôle excessif d'indicateurs trop nombreux. Les outils que nous mobilisons (carte causale et tableau de bord stratégique) visent, comme nous l'avons montré, à contrecarrer ces effets. (ii) Les approches de planification sont aussi interrogées par les théories de la décision qui montrent que les acteurs peuvent agir sans avoir de plan d'action prédéfini. Dans ce cas, à défaut de favoriser un pilotage de l'exploitation, notre itinéraire méthodologique a les vertus d'amener les acteurs à se poser des questions, à prendre du recul, à clarifier leur situation et à partager des directions. Ces vertus ne sont pas négligeables face à la complexité et utiles dans un contexte d'augmentation des formes sociétaires en agriculture qui s'accompagne d'une augmentation des tensions liée souvent à l'absence de projet partagé. (iii) Les critiques portent aussi sur la normativité du tableau de bord stratégique que proposent Kaplan et Norton (1996), arguant que ces auteurs proposeraient sans fondement et de manière quasi magique des indicateurs stratégique précédant les actions (Lorino, 2001). Nous dépassons cette critique par l'association de l'outil carte causale et du tableau de bord stratégique. Les objectifs traduits en indicateurs stratégiques proviennent d'une représentation de l'action vécue et de l'action projetée des agriculteurs. Ensuite, le processus vise à partir de l'indicateur stratégique à aller vers l'action à réaliser.

Enfin, l'itinéraire réclame quatre conditions de mise en œuvre. (i) La première est l'exigence, liée au recours à la carte causale et à un logiciel associé (Banxia/
Decision Explorer), de compétences de collecte, de traitement et de restitution structurée des représentations fidèles des agriculteurs. (ii) La deuxième est d'identifier et d'associer à la démarche le plus possible de parties prenantes de l'exploitation pour en construire une vision partagée. Cette condition a été vraie pour les exploitations d'établissements d'enseignement agricole public. Elle l'est également dans les exploitations de forme sociétaire ou unipersonnelle associant des collectifs de travail diversifiés. (iii) La troisième est que la réflexion stratégique exige un regard extérieur distancié, indépendant et non intéressé de la situation de l'exploitation afin d'éviter les biais cognitifs individuels (ancrage à un jugement initial, focalisation sur une solution, illusion de contrôle) (Laroche et al., 2006). Une structuration de la démarche autour de groupes d'agriculteurs et d'un travail collectif dans une logique de coconstruction, comme nous l'avons testé, devrait permettre d'atteindre ces résultats (Compagnone, 2009 ; Pervanchon et al., 2007). Le groupe de pairs est un lieu de mise en débat, de confrontation des idées, de production de connaissance et donc d'enrichissement des réflexions individuelles. (iv) La quatrième condition découle des précédentes : un accompagnateur est nécessaire (Hémidy et $a l ., 1996)$. Il doit être extérieur au groupe de réflexion. Il est garant de la bonne compréhension par l'ensemble des participants des processus de réflexion en jeu et doit faciliter les échanges (Von Korff et al., 2005). Ce type de prestation nous semble pouvoir être réalisé par l'appareil de formation, de conseil et d'appui aux agriculteurs et pris en grande partie financièrement en charge par les fonds de formation agricoles. Les deuxième et troisième conditions renvoient à une dimension collective de la démarche. Elle nécessite des compétences pour initier et animer le travail collectif. Les première et troisième conditions exigent des compétences techniques en termes de traitement 
de données et de mise en œuvre de l'itinéraire. Pour répondre à ces exigences, nous avons construit une formation destinée à des utilisateurs potentiels de l'itinéraire. La formation a été testée en 2012 en vue d'un déploiement plus large. Cette formation est proposée depuis 2013 aux conseillers agricoles et au personnel de l'enseignement technique agricole via une offre conjointe au catalogue de formation de Résolia, service de formation de l'assemblée permanente des Chambres d'agriculture, et au plan national de formation de l'enseignement agricole.

$$
\begin{gathered}
* \\
* *
\end{gathered}
$$

Comme dans les secteurs de l'industrie et des services, le management stratégique appliqué à l'exploitation agricole doit permettre aux agriculteurs de faciliter la conduite de leur exploitation dans un contexte changeant et perçu comme plus complexe. Il doit également leur permettre d'améliorer leurs performances.

Centré sur l'analyse que l'agriculteur fait de sa situation avec l'aide d'un conseiller et éventuellement au sein d'un groupe de pairs, l'itinéraire méthodologique PerfEA que nous proposons doit permettre aux responsables d'exploitations agricoles de s'inscrire dans une dynamique d'anticipation et d'amélioration continue à partir de la formalisation d'une stratégie et de sa traduction opérationnelle. Structuré en trois étapes, il aide à organiser la réflexion autour de la stratégie, puis à la formaliser dans un tableau de bord accompagné d'un plan d'action qui servira dans un troisième temps au pilotage de l'exploitation. Le lien entre ces trois étapes est assuré par la construction d'une carte causale. À l'instar des travaux de Lorino (2001), les parties prenantes de la stratégie de l'exploitation définissent selon quelles modalités d'action elles peuvent influer sur les résultats finaux et par quels outils d'information elles peuvent évaluer leurs actions.

Avec le développement de la contractualisation, de la certification environnementale des exploitations ou encore dans le cadre de la montée en puissance du développement durable et de la responsabilité sociétale des organisations, les démarches de management stratégique gagneraient à s'affirmer dans le secteur agricole comme un levier d'adaptation des agriculteurs aux évolutions rapides et incertaines de leur environnement. À ce titre, parce que l'itinéraire méthodologique proposé n'est pas nécessairement un outil dédié à une exploitation dont les limites sont fixées juridiquement ou économiquement, il pourrait, le cas échéant, servir à penser des stratégies collectives entre agriculteurs sur un territoire.

Nous remercions pour leur soutien financier, dans le cadre de la convention interrégionale de massif - Massif central, I'Union européenne via le Feder, l'État français par le Fnadt et la Région Auvergne. L'Ademe a aussi contribué au financementdeceprogramme. Nous remercions également les sept établissements publics locaux d'enseignement et de formation professionnelle agricoles $d u$ Massif central :Aurillac, Lempdes, Château-Chinon, Rodez, Figeac, La Canourgue et Limoges. 


\section{RÉFÉRENCES BIBLIOGRAPHIQUES}

Agreste (2011). Recensement agricole France Métropolitaine : Premières tendances. Agreste Primeur, $\mathrm{n}^{\circ}$ 266, p. 4.

Avenier M.-J., Schmitt C. (2008). Quelles perspectives le paradigme des sciences de l'artificiel offre-t-il à la recherche en management stratégique ? 9e CIFEPME/ Congrès International Francophone en Entrepreneuriat et PME, Louvain, Belgique, http://web.hec.ca/airepme/images/File/ 2008/C58.pdf, p. 22.

Axelrod R. (1976). Structure of decision. Princeton, New Jersey, Princeton University Press.

Barnard C. S., Nix J. (1979). Farm planning and control. Cambridge. New York, Cambridge University Press.

Barney J.-B. (1991). Firm resources and sustained competitive advantage. Journal of Management, vol. 17, $\mathrm{n}^{\circ}$ 1, p. 99-120.

Bossel H. (1999). Indicators for Sustainable Development: Theory, Method, Applications: a Report to the Balaton Group. Winnipeg, Manitoba, International Institute for Sustainable Development.

Brossier J., Chia E., Marshall E. (1988). Quelle gestion de trésorerie pour les agriculteurs? Gérer et Comprendre, Annales des Mines, $\mathrm{n}^{\circ} 12$, p. 41-55.

Brossier J., Chia E., Marshall E., Petit M. (1997). Gestion de l'exploitation agricole familiale : éléments théoriques et méthodologiques. Dijon, Enesad/Cnerta.

Casavant K. L., Infanger C. L. (1999). Agricultural Economics \& Management. Upper Saddle River - New Jersey, Prentice Hall.

Chabin Y. (2001). La cohérence entre représentations de la performance et contrôle : le cas des entreprises intégrées de grande distribution alimentaire. Montpellier, Université des sciences et techniques de Montpellier 2. Thèse de doctorat en sciences de gestion, p. 422.

Chabin Y. (2008). Pilotage et mesure de la performance stratégique en cave coopérative. Colloque SFER Les entreprises coopératives agricoles, mutations et perspectives, atelier E2, Paris, p. 11.

Charbonnier E., Cathala A., De Smedt H. (2009). Formation : mon groupe dans son environnement. Trame et Cabinet Cohéliance, p. 1-5

Chombart de Lauwe J., Poitevin J. Tirel J.-C. (1969). Nouvelle gestion des exploitations agricoles. Paris, Dunod.

Compagnone C. (2009). Conseil collectif et collectifs de production de connaissances. In Compagnone C., Auricoste C., Lémery B. (Eds.), Conseil et développement en agriculture. Quelles nouvelles pratiques? Dijon, Educagri, Éditions Quae, p. 19-35.

Cossette P. (1994). Cartes cognitives et organisation. Laval, Presses de l'Université de Laval.

Cossette P. (2003). Méthode systématique d'aide à la formulation de la vision stratégique : illustration auprès d'un propriétaire dirigeant. Revue de l'entrepreneuriat, vol. 2, $\mathrm{n}^{\circ} 1$, p. 18.

David A. (2000). La recherche-intervention, cadre général pour la recherche en management ? In David A. H. R., Laufer R (Eds.), Les nouvelles fondations des sciences de gestion, Paris, Vuibert, p. 193-213.

Dedieu B., Laurent C., Mundler P. (1999). Organisation du travail dans les systèmes d'activités complexes. Économie rurale, $\mathrm{n}^{\circ} 253$, p. 28-35.

Eden C. (1988). Cognitive Mapping-A Review. European Journal of Operational Research, $\mathrm{n}^{\circ} 36$, p. 1-13.

Fairweather J. (2010). Farmer models of socioecologic systems: Application of causal mapping across multiple locations. Ecological Modelling, vol. 221, $\mathrm{n}^{\circ} 3$, p. 555-562.

Goulet F., Pervanchon F., Conteau C., Cerf M. (2008). Les agriculteurs innovent par euxmêmes pour leurs systèmes de culture. In Reau R., Doré T. (Eds.), Systèmes de culture innovants et durables, Dijon, Educagri, p. 53-69. 
RECHERCHES

Guichard M., Michaud R. (1994). La stratégie à pas contés : piloter l'entreprise agricole dans l'incertitude et dans la complexité. Dijon, Enesad/Cnerta-SED.

Hémidy L., Boiteux J., Cartel H. (1996). Aide à la décision et accompagnement stratégique : l'expérience du CDER de la Marne. Communication pour le colloque INRA/Pour la terre et les Hommes, 50 ans de recherches à l'INRA, Laon, p. 20.

Hémidy L., Maxime F., Soler L.-G. (1993). Instrumentation et pilotage stratégique dans l'entreprise agricole. Cahiers d'économie et sociologie rurales, $\mathrm{n}^{\circ} 28$, p. 91-118.

Hémidy L., Soler L.-G. (1994). Un modèle conceptuel pour le pilotage stratégique de l'entreprise agricole. Communication pour le $38^{\mathrm{e}}$ séminaire EAAE, 3 au 5 octobre 1994, Copenhague, p. 14.

Huff A. (1990). Mapping Strategic Though. New York, Wiley \& Sons.

Jeanneaux P., Blasquiet-Revol H. (2012). La gestion de 1'exploitation agricole : un état des lieux des recherches françaises. Gérer et Comprendre, $\mathrm{n}^{\circ} 107$, p. 29-40.

Jérome L.-P. (2001). Résoudre des problèmes et produire des idées. La technique du brainstorming. Travaux \& Innovations, $\mathrm{n}^{\circ} 83$, p. 9-12.

Kaplan R., Norton D. (1996). Using the Balanced Scorecard As a Strategic Management System. Harvard Business Review, $\mathrm{n}^{\circ}$ 1996-1, p. 75-85

Kaplan R., Norton P. (2004). Strategy Mapsconverting intangible assets into tangible outcomes. Boston, Massachusetts, Harvard Business School Press.

Kay R. D. (1986). Farm management : planning, control, and implementation. New York, McGraw-Hill.

Laroche H., Nioche J.-P. (2006). L'approche cognitive de la stratégie d'entreprise. Revue française de gestion, $\mathrm{n}^{\circ} 160$, p. 81-105.

Laukkanen M. (1998). Conducting Causal Mapping Research: Opportunities and Challenges. In Eden C., Spender J.-C. (Eds.), Managerial and Organizational CognitionTheory, Methods and Research, London, Sage Publication, p. 168-191.
Laurent C. (2000). La multifonctionnalité de l'agriculture. In Durand M.-F., Giordano P. Valladao A. (Eds.), Towards an agreement between Europe and Mercosur, Paris, Presses de Sciences Politiques.

Lérat-Pytlak J. (2002). Le passage d'une certification ISO 9001 à un management par la qualité totale. Toulouse, Université des sciences sociales, Toulouse I. Thèse de Doctorat en Sciences de Gestion, p. 471.

Lorino, P. (2001). Le Balanced Scorecard revisité : dynamique stratégique et pilotage de performance. http://halshs.archives-ouvertes.fr/docs/00/58/46/37/PDF/LORINO. PDF, 22 $2^{\mathrm{e}}$ congrès de l'AFC, p. 20.

Marshall E., Bonneviale J.-R., Francfort I. (1994). Fonctionnement et diagnostic global de l'exploitation agricole. Une méthode interdisciplinaire pour la formation et le développement. Dijon, Enesad-Sed.

Mazé A., Aubry C., Papy F. (2000). La certification des exploitations agricoles. Économie rurale, $\mathrm{n}^{\circ} 258$, p. 134-139.

Mellet, S. (2007). Expérimentation Système de Management Environnemental-ISO 14001. Application au secteur agricole en région Rhône-Alpes. Rapport technique 20032006. Lyon, DGER, DRAF Rhône-Alpes SRFD, p. 47.

Mignolet C., Fiorelli J.-L., Peyre D., Danguy des Deserts F., Georgel R. (1998). L'agrandissement des exploitations d'élevage dans la plaine des Vosges : quelles répercussions pour la durabilité des systèmes de production? $5^{\text {es }}$ Rencontres Recherches Ruminants, Paris, p. 161-164.

Mintzberg H. (1994). The Rise and Fall of Strategic Planning: Reconceiving the Roles for Planning, Plans, Planners. New York, The Free Press.

Noell C., Lund M. (2002). The balances Scorecard (BSC) for Danish Farm-Vague framework or functional instrument. Farm management Proceedings of NJF Seminar, $\mathrm{n}^{\circ} 345$, Oslo, p. 187-204.

Olson K. D. (2004). Farm Management: Principles and Strategies Iowa. Blackwell Publishing Co. 


\section{Management stratégique des exploitations agricoles}

Pervanchon F., De Torcy B., Delépine F.-X. (2007). Dossier Formation : Autonomie de décision et démarche stratégique. Travaux \& Innovations, $\mathrm{n}^{\circ} 140$.

Rattin S. (2007). Les sociétés agricoles en pleine croissance. Agreste cahiers Spécial $R A 2000, \mathrm{n}^{\circ} 2$, p. $49-56$.

Séronie J.-M., Boullet P. (2007). L'exploitation agricole flexible. CER France, Les Cahiers du CER, p. 36.

Shewhart W. (1989). Les fondements de la mấtrise de la qualité. Paris, Economica.

Simon H. (1982). Models of Bounded Rationality. Boston, MIT Press.

Simon H. (2004). Les sciences de l'artificiel. $1^{\text {reo }}$ édition en 1969, Paris, Gallimard.
Thiétart R.-A. (2004). Comprendre l'entreprise et son organisation. Les composantes du management. Cahiers français, ${ }^{\circ} 321$, p. 3-8.

Trémolet, P. (2008). Picardie : ils osent l'Iso 14001. Gérer pour gagner en agriculture, $\mathrm{n}^{\circ} 10$, p. 3.

Turner J., Taylor M. (1998). Applied farm management. Oxford, Blackwell Science.

Vernerey G. (2007). Conseils pour développer des formations à la stratégie. Travaux \& Innovations, $\mathrm{n}^{\circ} 140$, $\mathrm{p}$. XXVI.

von Korff Y., Guetta I. (2005). Description du travail de facilitateur professionnel. http:// www.iaf-world.org/files/public/IAF_Fr_ description_IG.3_doc.pdf. 